\title{
Application of Internet of Things in the Field of Medical and Smart Health Care: A Review
}

\author{
Diksha B. Wasankar ${ }^{1}$, Dr. Vijay S.Gulhane ${ }^{2}$, L. K. Gautam ${ }^{3}$ \\ ME Scholar, Dept of Computer Science and Engg, Sipna Collage of Engineering and Technology, Amravati, India ${ }^{1}$ \\ Professor, Dept of Information Technology, Sipna Collage of Engineering and Technology, Amravati, India ${ }^{2}$
}

Assistant Professor, Dept of Information Technology, Sipna Collage of Engineering and Technology, Amravati, India ${ }^{3}$

\begin{abstract}
IOT basically comprises of physical articles that are installed with sensors, actuators, processing gadgets and information correspondence abilities. These are connected to systems for information transportation. Envision a situation where patient's restorative profile, indispensable parameters, and dialysis machine info are caught with the assistance of medicinal gadgets connected to his body. The patient does not need to move from office to office to get treatment. Or maybe, he can complete his dialysis the assistance of a versatile home machine intended for the reason. Information assembled from this gadget is broke down and put away, and the accumulation from different sensors and restorative gadgets settles on educated choices in convenient way. Parental figures can screen the patient from any area and react properly, in light of the caution got. Propelled treatment of this nature can radically enhance a patient's personal satisfaction. The current headways in innovation and the accessibility of the Web make it conceivable to interface different gadgets that can speak with each other and share information. The Web of Things (IoT) is another idea that permits clients to interface different sensors and savvy gadgets to gather continuous information from nature. The model named as 'k-Social insurance' makes utilization of 4 layers; the sensor layer, the system layer, the Web layer and the administrations layer. All layers collaborate with each other viably and proficiently to give a stage to getting to patients' wellbeing information utilizing advanced mobile phones. The quick development of electronic gadgets, advanced mobile phones and tablets which can be imparted physically or remotely has turned into the principal device of day by day life. The up and coming era of associated world is Web of Things (IoT) which interfaces gadgets, sensors, machines, vehicles and other "things".
\end{abstract}

Keywords: RFID, IPv6, Cloud computing.

\section{INTRODUCTION}

With the assistance of IoT, we interface anything, access from anyplace and at whatever time, and productively get to any administration and data about any question. The point of IoT is to develop the advantages of Web with remote control capacity, information sharing, steady network et cetera. The term IoT, frequently called Web of everything, was first presented by Kevin Ashton in 1999 who dreams a framework where each physical question is associated utilizing the Web through pervasive sensors. The IoT innovation is these days utilized as a part of various fields of life including advanced oilfield, home and building mechanization, savvy Lattice, computerized restorative treatment, astute transportation and so on.

\section{LITERATURE REVIEW}

RFIDs utilize the radio recurrence labels to distinguish genuine items, and RFID sensor exchanges information between as per user and a question which is recognized track and arrange. RFID can utilize two distinct sorts of labels: Dynamic and Latent. IPv6 and Distributed computing advance the improvement of incorporation of Web and IoT. Information is handled and put away in the distributed storage. The put away information can be gotten to by professionals and restorative staff later on to watch and screen patient's wellbeing. It is giving more potential outcomes of information gathering, information preparing, port administration and other new administrations. Each question which associates with IoT requires a one of a kind address or recognizable proof which can be refined with the assistance of IPv6.

Usage of the Web of Things is adaptable and open outcomes to permitting the social insurance applications to serve patients with better treatment, likewise finished with the remote patient checking and powerful restorative information dealing with. There are some of various qualities are expected to actualize the medicinal services benefit in the earth.

1) Ontology Assessment

In this part sources of info are changed over into capacity in which patients indications are investigated, infections are found, and all the data's are put into the remote database. At the point when the patient first enters the clinics, the 
physical attributes of the illnesses will be essentially dictated by the specialist. The decided capacities are partitioned into the classes and sub classes. Classes are speaks to the patients essential data and subclasses are speaks to definite ailments data.

\section{2) Global Philosophy Correlation}

In this assessed philosophy contrasted with the all inclusive put away ailments cosmology on the base of the learning base framework. Worldwide cosmology contains the two types of metaphysics; these are infection philosophy and asset metaphysics. Malady metaphysics contains the patient essential and therapeutic data and asset cosmology covers restorative assets to such an extent that specialists, medicinal gadgets, and so on.

\section{3) Similarity Counts}

Input the request substance of the manifestation physically. Contrast the information indication and all the worldwide infection philosophy in the learning base. Perform comparability coordinating of the side effects consequently for closeness coordinating. Computerize the choice of the most comparable case in the information base. It is anything but difficult to discover what sorts of gadgets are required for the comparing side effects and restoration techniques through the metaphysics.

\section{4) Design Enhancement}

The last period of savvy configuration prepare in which the technique, strategies, degrees, and length of the majority of the different activities. Parameter enhancement is basic to make guaranteed that the particulars run over the prerequisites from structure. Programmed plans help to manufacture a framework where another patient could be immediately analyzed, relating help system can soon be worked out, and related therapeutic properties can be dispersed in a brief timeframe. With metaphysics guaranteeing the all around requested information structure [1].

[2] Sensors can give data about the Physical Substance they screen. Data in this setting ranges from the personality to measures of the physical condition of the Physical Substance. The personality can be innately bound to that of the gadget, as on account of implanted gadgets, or it can be gotten from perception of the question's elements or connected Labels. Inserted Sensors are joined or generally installed in the physical structure of the Physical Element with a specific end goal to improve and give guide association with other Keen Protests or to the system. Subsequently they likewise recognize the Physical Substance. Sensors can likewise be outer gadgets with locally available sensors and complex programming which typically watch a particular situation in which they can distinguish and screen Physical Elements, using complex calculations and programming preparing strategies. The most widely recognized case of this classification is face acknowledgment frameworks which utilize the optical range.

[3] We examined the significant reviews revealed in the referenced papers, we see that some of specialists proposed new designs and models for IoT, which help to convey IoT in the field of medicinal and social insurance. It is likewise seen that a portion of the creators take after IEEE and different principles to execute their proposed IoT model to give remote checking and crisis help while a portion of the creators basically clarify the uses of IoT in medicinal services.

We assess the proposed IoT models in light of a few parameters, for example, arrangement of crisis help, innovation utilized, standard took after, support for multi gadget and manmade brainpower usage.

Crisis Help: Utilizing IoT in the field of therapeutic and social insurance, the attention ought to be on information and on the arrangement of the support in crises. The framework must create alerts, advise the patients and experts.

Innovation: IoT bolsters distinctive and most recent advances like RFID, WSN, 3G, 4G systems and so forth. Utilizing these innovations, one can get information identified with patient's wellbeing and send it to a remote server for further handling and capacity.

Gauges: IoT bolsters diverse benchmarks and conventions, e.g. IEEE 802.11/b/g/n, IEEE 802.15.4, IEEE 802.15.6, ZigBee, WBAN, and NL 7 and so on. Utilizing benchmarks and conventions, we can discover the separation, exactness and time to take a framework to finish his work.

Multi gadget bolster: We can look at changed models and frameworks on the bases of multi gadget bolster. The productive frameworks bolster numerous gadgets, for example, RFID sensors, body sensors, advanced mobile phone sensors, tablets, and wearable gadgets.

[4] In crisis medicinal administrations, to enhance the nature of human services administrations, conveying center data of patient at the purpose of-care to doctors is basic. In any case, restorative records and facility information are put away in various healing centers, it is once in a while hard to accumulate facility information of patient pervasively if 
there should be an occurrence of crisis. So as to proceed with the omnipresent substance getting to, this paper proposed an asset model to find and get center information which are put away in heterogeneous doctor's facility data frameworks. At that point, omnipresent information getting to strategy is presented in light of the asset display. In the new technique, facility information of patient is characterized as asset with exceptional URL address. Related facility information of one patient is assembled to shape a collected asset, and could be associated by doctor if expert is allocated to the doctor. At last, contextual analysis is talked about to clarify the strategy for facility information getting to through Web from various human services units. The outcome demonstrates that the patient's record could be gotten to all the more advantageously.

In medicinal services benefit, specialists, patients, doctors assume a noteworthy part and they additionally included in a whole overhauling. Specialists need to get to the patient record from anyplace by putting away it in an appropriated way. Patients likewise needs to about the specialists accessibility and the types of gear status (occupied/free). Keeping in mind the end goal to help persistent getting to specialist's accessibility status, an asset model is required for this availability.

[5] The present development of the customary therapeutic model toward the participatory pharmaceutical can be helped by the Web of Things (IoT) worldview including sensors (ecological, wearable, and embedded) spread inside household situations with the reason to screen the client's wellbeing and initiate remote help. RF distinguishing proof (RFID) innovation is currently develop to give some portion of the IoT physical layer for the individual medicinal services in savvy conditions through minimal effort, vitality self-governing, and expendable sensors. It is here displayed a study on the best in class of RFID for application to body driven frameworks and for social event data (temperature, moistness, and different gasses) about the client's living condition. Numerous accessible alternatives are portrayed up to the application level with a few cases of RFID frameworks ready to gather and process multichannel information about the human conduct in consistence with the power introduction and sterile directions

[6] The IoT assumes a huge part in a wide scope of medicinal services applications, from overseeing endless maladies toward one side of the range to forestalling illness at the other. Here are a few cases of how its potential is as of now playing out:

Clinical care: Hospitalized patients whose physiological status requires close consideration can be always observed utilizing IoT-driven, noninvasive checking. This kind of arrangement utilizes sensors to gather far reaching physiological data and utilizations entryways and the cloud to investigate and store the data and afterward send the broke down information remotely to guardians for further examination and survey. It replaces the way toward having a wellbeing proficient stop by at customary interims to check the patient's imperative signs, rather giving a consistent mechanized stream of data. Thusly, it at the same time enhances the nature of care through steady consideration and brings down the cost of care by wiping out the requirement for a guardian to effectively participate in information gathering and investigation.

Remote observing: There are individuals everywhere throughout the world whose wellbeing may endure on the grounds that they don't have prepared access to powerful wellbeing checking. Be that as it may, little, effective remote arrangements associated through the IoT are presently making it workable for observing to go to these patients rather than the other way around. These arrangements can be utilized to safely catch understanding wellbeing information from an assortment of sensors, apply complex calculations to break down the information and after that impart it through remote availability to therapeutic experts who can make fitting wellbeing suggestions. A case of an empowering innovation for remote observing is the Freescale Home Wellbeing Center point reference stage, which is based on Freescale i.MX applications preparing innovation and firmly coordinates key capacities, for example, remote availability and power administration-in the telehealth portal that empowers accumulation and sharing of physiological data. The center point catches persistent information from an assortment of sensors and safely stores it in the cloud, where it can be gotten to by those occupied with the patient's care.

[7] Associated medicinal services programs use rare assets to give an enhanced nature of care, prompting better clinical results. Quantifiable advantages of associated restorative gadgets incorporate lessening in death rates, diminished center visits, crisis confirmations, and healing facility affirmations, incorporating decrease in bed days of care and length of remain in clinics. The significant utilize cases in this space include:

Watch over the pediatric and the matured: Associated wellbeing projects are intended to enhance existing social insurance frameworks for pediatric and maturing populaces. The key thought is to permit the care to be managed in the patient's home. Pediatric patients require consideration and particular care. Most matured patients, then again, have various perpetual illnesses. Their physiological parameters, exercises and home condition require consistent 
supervision. Another class of gadgets distinguishes inclination and feeling which is utilized to screen the mental condition of the patient.

Interminable infection administration: Incessant sicknesses, for example, cardiovascular ailment, diabetes, elevated cholesterol; hypertension, corpulence, aspiratory issues and different illnesses influence billions of individuals around the world. Ceaseless ailment administration includes dealing with the way of life of the patient through persistent engagement, to help the patient hold fast to a customized mind arrange including particular treatment, pharmaceutical and eating regimen administrations.

Personal health and fitness management: This class of applications is intended for people who are self motivated and take steps to stay healthy and fit. These users monitor their daily exercise and fitness regimes and use an app or web based application to store the data and keep track of their progress. These apps are also used to track adherence to schedules created by trainers. Sensors and devices used in this case include weight sensors, activity monitors, heart rate and blood pressure monitors, connected treadmills and other fitness equipment.

\section{III.PROPOSED WORK}

We can construct systems which can continuously monitor the patients, remote consultation, critically-ill patients and health care management platform using different techniques and equipment which can sense, capture, measure and transmit the information of body or things. Combining sensors and the microcontroller to get accurate measurement, and monitoring and analyzing the health status increase the power of IoT in healthcare. These can include blood pressure, heart rate, oxygen saturation in blood, levels of glucose and motion of body.

Evaluate the proposed IoT models based on some parameters such as provision of emergency aid, technology used, standard followed, support for multi device and artificial intelligence implementation.

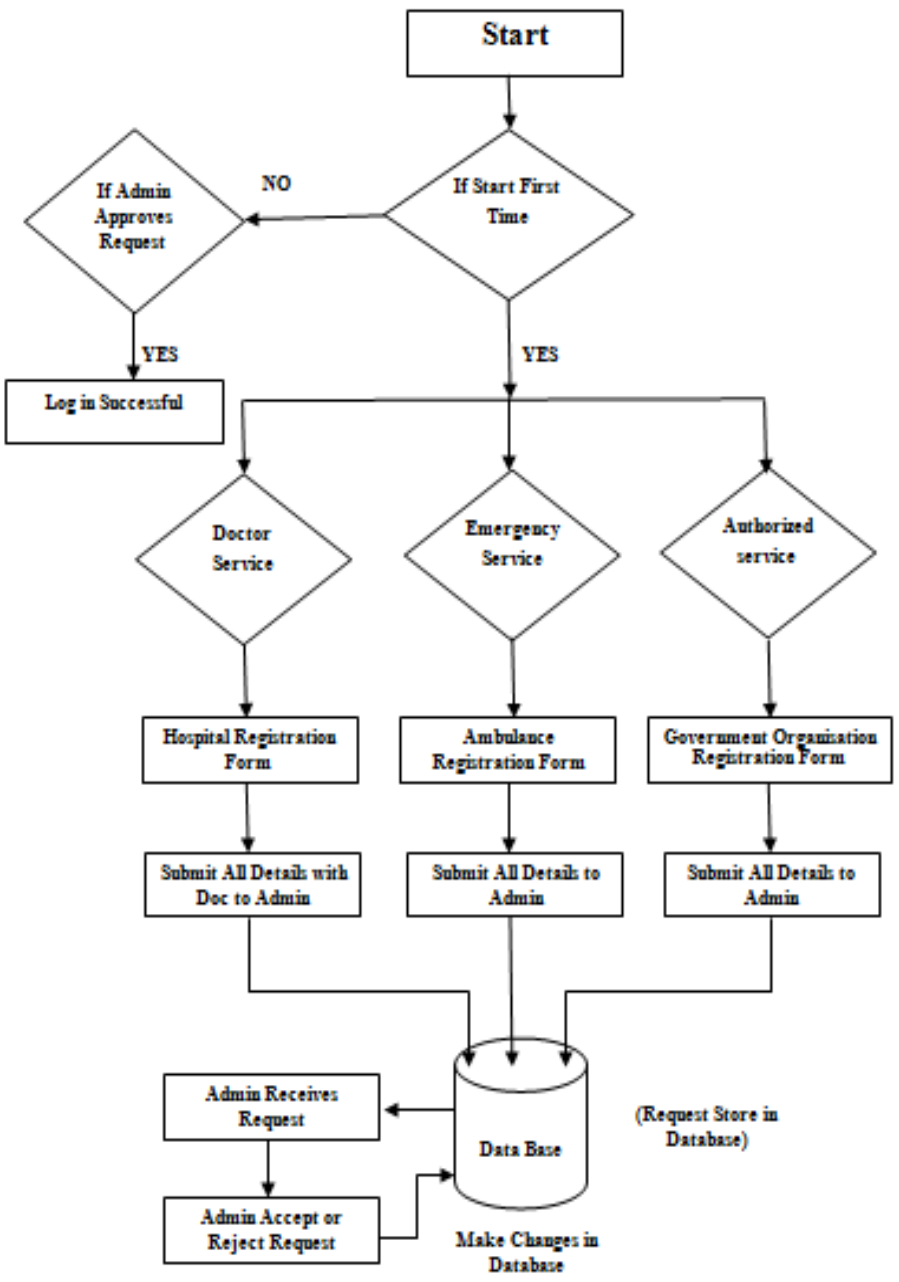

Fig 1 basic block diagram of application smart health care 


\section{A. Emergency Aid}

Using IoT in the field of medical and healthcare, the focus should be on data and on the provision of the support in emergencies. The system must generate alarms, inform the patients and consultants.

\section{B. Technology}

IoT supports different and latest technologies like RFID, WSN, 3G, 4G networks etc. Using these technologies, one can obtain data related to patient's health and send it to a remote server for further processing and storage.

\section{Standards}

IoT supports different standards and protocols. Using standards and protocols, we can find the distance, accuracy and time to take a system to complete his work.

D. Multi device support

We can compare different models and systems on the bases of multi device support. The efficient systems support many devices such as RFID sensors, body sensors, smart phone sensors, tablets, and wearable devices.

\section{REFERENCES}

[1] P. Elanthiraiyan, Dr. S. Babu 'Smart Medicine and Physical Health System Using IoT' International Journal of Computer Science and Mobile Computing, Vol.4 Issue.3, March- 2015.

[2] Alexandru Serbanati, Carlo Maria Medaglia and Ugo Biader Ceipidor "Building blocks of IOT:state of the art and beyond" CATTID"Sapienza" University of Rome Italy.

[3] Kaleem Ullah, Munam Ali Shah, Sijing Zhang "Effective Ways to Use Internet of Things in the Field of Medical and Smart Health Care". 9781-4673-8753-8/16/2016 IEEE.

[4] Abinaya, Raja Sekar "HealthCare Information System for Emergency Medical Service based on Ubiquitous Data Accessing - Internet of Things. KJER Volume No.: II, Special Issue on IEEE Sponsored International Conference on Intelligent Systems and Control (ISCO'15).

[5] K. Natarajan, B. Prasath, P. Kokila "Smart Health Care System Using Internet of Things" Journal of Network Communications and Emerging Technologies (JNCET) Volume 6, Issue 3, March (2016).

[6] "How the Internet of Things Is Revolutionizing Healthcare" freescale.com/healthcare Document Number: IOTREVHEALCARWP REV October 2013.

[7] Ashok Khanna, Prateep Misra "The internet of things for medical devices prospects, challenges, and the way forward". TCS.comRSS:http://www.tcs.com/rss_feeds/Pages/feed.aspx?f=wFeedburner:http://feeds2.feedburner.com/tcswhitepapers. 